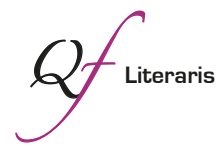

\title{
Traducción, censura y construcción del discurso literario. La labor de los editores J. Janés, C. Barral y J. M. Castellet durante el franquismo
}

\author{
Jordi Jané-Lligé \\ Universitat Autònoma de Barcelona \\ Jordi.Jane@uab.cat
}

Resumen: Traducción y censura tuvieron un papel primordial en la articulación de la vida cultural y literaria durante el franquismo, en este artículo se valoran recientes aportaciones de la traductología para abordar sus relaciones. De un lado se considera críticamente el término textualidad nacional, propuesto por Francesca Billiani, y de otro lado se destaca la distinción entre las alteraciones producidas en los textos traducidos por la censura de aquellas motivadas por otras convenciones literarias, propuesta por Rundle y Sturge. Finalmente se resalta la labor de tres editores: Josep Janés, Carlos Barral y Josep Maria Castellet en la renovación del panorama editorial español y catalán a través de la traducción y se describen sus relaciones con la censura.

Palabras clave: traducción; censura; textualidad nacional; mundo editorial; convenciones literarias.

\begin{abstract}
Translation and censorship played a central role in shaping literary and cultural life during Francoism. In this paper I evaluate recent contributions to the analysis of their relationship. On the one hand I consider critically Billani's concept of "textual nationality", on the other hand the distinction in translated texts made by Rundle and Sturge between alterations due to the intervention of censorship and those due to literary conventions or matters of good taste. Lastly I emphasize the work by three editors: Josep Janés, Carlos Barral and Josep Maria Castellet in renewing the Spanish and Catalan publishing landscape through translations and their relationship to censorship.
\end{abstract}

Keywords: translation; censorship; textual nationality; publishing world; literary conventions.

》 Jané-Lligé, Jordi. 2015. "Traducción, censura y construcción del discurso literario. La labor de los editores J. Janés, C. Barral y J. M. Castellet durante el franquismo". Quaderns de Filologia: Estudis Literaris XX: 73-90. doi: 10.7203/ qdfed.20.7529 



\section{Introducción}

Pero hay todavía otro aspecto de la misma cuestión, al que aludimos en el capítulo 5 y del que hemos de hablar nuevamente. Nos referimos al clamor levantado cerca de la opinión pública por una parte de nuestra prensa contra el pretendido exceso de traducciones y al intento que se vislumbra de tratar de poner coto a tal exceso interviniendo el número de traducciones e imponiendo una tasa (Gili, 1944: 112).

Con estas palabras escritas en el año $1944^{1}$ alude el editor barcelonés Gustau Gili Roig a ciertas voces de alarma aparecidas durante los primeros años de la posguerra ante la progresiva recuperación del ritmo de publicación de traducciones en el marco de la producción editorial española una vez concluida la contienda civil ${ }^{2}$. Gili Roig, en su estudio, aporta cifras sobre la totalidad de libros publicados anualmente en España entre 1928 y 1944, y respectivamente del número de traducciones, y ofrece el cálculo del porcentaje que representan estas con relación al total. Así, los títulos traducidos en España durante los primeros años cuarenta significan aproximadamente el $25 \%$ del total de la producción editorial (en 1943 el 25,54\%), acercándose paulatinamente de este modo a los porcentajes a los que se había llegado en los años anteriores a la Guerra Civil (en 1928 representaban un 24\%, y en el año de máxima actividad traductora de los analizados, el año 1931, un 36,7\%). Con sus datos y comentarios Gili Roig no solo intenta quitar hierro al asunto, sino que hace una defensa a ultranza de la necesidad de la traducción en cualquier sociedad tanto desde el punto de vista cultural como del económico y empresarial. El editor barcelonés, a sus ya 75 años, afirma con valentía:

\footnotetext{
* Este artículo se inscribe en el Grup d'Estudi de la Traducció Catalana Contemporània (GETCC) (2014, SGR 285), reconocido por la Agència de Gestió i Ajuts Universitaris de la Generalitat de Catalunya, y en el proyecto "La traducción catalana contemporánea: censura y políticas editoriales, género e ideología (1939-2000)”, con el número de referencia FFI2014-52989-C2-1-P, financiado por el Ministerio de Economía y Competitividad.

${ }^{1}$ Gustavo Gili Roig (1944) Bosquejo de una política del libro.

${ }^{2}$ Jacqueline Hurtley (1992: 158-163) lo describe con detalle en su estudio sobre la labor de Josep Janés como editor de literatura inglesa. Las voces críticas correspondían a personalidades vinculadas a organismos oficiales o a algunos rotativos de la prensa diaria.
} 
Así pues, acaso sería saludable que algunos de los más apasionados comentaristas, al enjuiciar esta cuestión, tuvieran en cuenta que no siempre la autarquía intelectual es sinónimo de imperio (Gili, 1944: 36).

El editor reconoce asimismo que en determinados ámbitos editoriales los porcentajes llegan a dispararse. Así, comenta Gili Roig:

Una parte importante de las traducciones que se publican en España corresponde a la literatura en general, y especialmente al género novelístico. En 1942, sobre un total de 1242 obras de literatura, aparecieron 527 traducciones, o sea el 42,4 por 100 (Gili, 1944: 36).

No obstante, el editor califica esta realidad en los siguientes términos:

[...] actividad traductora, que algunos interpretan como desgana del lector español por lo español y que nosotros estimamos como un índice excelente de nuestra curiosidad colectiva y nuestro afán de superarnos importando lo mejor que produzcan los extranjeros [...] (Gili, 1944: $38)$.

Si a los ojos de un veterano editor, formado en épocas de mayor liberalidad, estos porcentajes eran más que deseables en todos los sentidos, no es sorprendente que no se lo parecieran tanto a las instancias culturales oficiales del franquismo. Pues de entrada parecería lógico que un régimen dictatorial fuertemente vinculado en sus inicios al fascismo, y por ende a una determinada visión regeneradora de la nación, que se caracterizaba por la exaltación de la patria y sus esencias, y que tenía a su disposición todos los mecanismos de difusión y también de control existentes, hubiese logrado sentar las bases de un modelo cultural sólido y genuino, capaz de seducir a amplios sectores de la población.

Nada más lejano de la realidad. Tal como se reitera en la abundante bibliografía existente al respecto ${ }^{3}$, el franquismo no supo crear a lo largo su dilatada existencia un discurso cultural consistente y atractivo, que despertara el interés de la mayoría de los españoles. Una de las consecuencias de ello fue que buena parte de los productos culturales que se consumieron en España durante ese período -también y espe-

\footnotetext{
3 Jacqueline Hurtley (1992), José Martínez Cachero (1997), Jordi Gracia \& Miguel Ángel Ruiz Carnicer (2001), Pere Ysàs (2004) y Santos Sanz Villanueva (2010), por citar solo algunos ejemplos.
} 
cialmente los más populares, como el cine y la novela de entretenimiento- procedieron de la importación. De este fiasco ${ }^{4}$ dan fe, por poner dos ejemplos en cierto modo opuestos, tanto las andanzas de iniciativas editoriales estatales como la Editora Nacional, profusamente estudiada en su etapa inicial por Eduardo Ruiz Bautista (2005: 187-240), como la díscola actividad de traducción llevada a cabo frenéticamente por editores como Josep Janés en los primeros años del franquismo, tal como documentan Jacqueline Hurtley (1986; 1992) y Josep Mengual (2013), o en etapas posteriores Carlos Barral (Castellet, 2001) y, en el ámbito de la traducción catalana, Josep Maria Castellet (Muñoz Lloret, 2006) -en el caso de Castellet, una vez levantado el veto institucional a publicar traducciones al catalán a principios de los años sesenta.

Las razones que puedan explicar tanto la ineficacia de las políticas culturales activas (que no las reactivas) durante el franquismo, como la necesidad de una importación masiva de bienes culturales en España durante la dictadura son sin duda alguna complejas y de naturaleza muy diversa. La aportación específica que pueden ofrecer los estudios de traducción y recepción al mejor conocimiento de este período de la historia cultural en España debe incidir básicamente en el estudio de las iniciativas editoriales de traducción que se llevaron a cabo en las diferentes etapas del franquismo y del trato que la censura les dispensó, de un lado, y de otro, en el análisis del impacto y los efectos que estas tuvieron en la vida cultural y literaria españolas.

\section{2. "Textualidad nacional" (Billiani); "Censura/ convenciones literarias/ buen gusto" (Rundle/ Sturge)}

El creciente interés que cabe acreditar en todo el mundo por el estudio de las relaciones entre censura y traducción en diferentes contextos culturales e históricos ${ }^{5}$ responde sin duda alguna entre otras cosas a la relativamente reciente incorporación a los estudios de traducción de los

\footnotetext{
${ }^{4}$ Ya Gustau Gili (1944: 101) escribía acerca del primer franquismo: "En un régimen que interviene todas las publicaciones, como el actual, parecería oportuno que el Nuevo Estado se hubiese ocupado en imponer unas consignas claras y terminantes en favor de nuestro libros, que es lo mismo que decir para la expansión de nuestra cultura [...]". ${ }^{5}$ Además de Billiani (2007) cabe destacar las monografías que dedican al tema la revista Quaderns. Revista de traducció, n. 20 (2013); Teresa Seruya \& Maria Lin (2008) y Robert C. Post (1998) además de un sinnúmero de artículos.
} 
enfoques sociológico e ideológico (Prunč, 2012: 315-334), con sus diferentes planteamientos y parámetros de análisis (Inguileri, 2005: 125$145)^{6}$. En los contextos políticos donde existe (o existió) una censura institucional (asociada habitualmente a un régimen político no democrático), el análisis de la actividad traductora y su regulación por parte de los organismos oficiales sacan a la luz de una forma muy ilustrativa y esclarecedora los componentes ideológico, político y sociológico que sin duda alguna caracterizan la producción cultural. En sus últimos trabajos dedicados al estudio de la traducción y la censura, muy centrados en el contexto de los fascismos europeos, Francesca Billiani (2007), de un lado, y Christopher Rundle y Kate Sturge (2010), de otro, proponen nuevas herramientas de análisis que cabe tomar en consideración para el estudio de la traducción y la censura durante el franquismo.

\section{1. "Textualidad nacional"}

Francesca Billiani recurre al término textualidad nacional ${ }^{7}$ para describir, de un lado, la toma de conciencia que la actividad traductora trae consigo, por contraste con otras tradiciones, acerca de la existencia de una tradición textual nacional, y de otro lado, para determinar la forma en que la censura actúa al reclamar alteraciones en los textos a traducir en nombre del respeto a la naturaleza de esa "textualidad nacional", acorde, por supuesto, con los principios ideológicos y estéticos dominantes del régimen imperante.

A pesar de que el término textualidad nacional no parece desacertado para dotar de sentido a los veredictos con que la censura legitima la condena parcial o absoluta de un buen número de autores y obras, ese concepto presenta algunos problemas en su aplicación, que nosotros veremos concretados en el estudio de la censura en España. De forma general, y eso es válido no solo para la censura española, puede decirse que la censura no fue, no puede ser, un organismo o una actividad homogénea, y por tanto parece poco factible hablar de una "textualidad nacional" susceptible de ser definida unívocamente. Centrados en el

\footnotetext{
${ }^{6}$ Inghilleri señala especialmente la influencia de la obra de Pierre Bourdieu y de Niklas Luhmann en las distintas aproximaciones existentes en la actualidad en los estudios de traducción que cabe relacionar con el enfoque sociológico.

${ }^{7}$ Billiani toma prestado el término de Homi Bhabha, tal como refiere en su estudio sobre censura y traducción (2007: 16).
} 
análisis concreto de la censura española cabe destacar de entrada tres elementos que distorsionan cierta visión compacta del hecho censor: en primer lugar, la larga vida del franquismo; en segundo lugar, la realidad pluricultural de España, que dada su vitalidad y riqueza se distingue manifiestamente de otras realidades europeas; en tercer lugar, la patente opacidad de la institución censora en las actuaciones de sus distintas jerarquías (los lectores y autores de informes de censura, los responsables políticos, los responsables religiosos).

La censura franquista (y sus "principios de textualidad nacional") estuvo sujeta a numerosos cambios de orientación de acuerdo con los vaivenes políticos e ideológicos por los que el franquismo atravesó tanto interna como internacionalmente durante toda su andadura ${ }^{8}$, como lo demuestra claramente el trato cambiante e incluso contradictorio que dio a ciertos autores ${ }^{9}$ y obras. Además, esa "textualidad nacional" se definió en España antes que nada y principalmente como negación de otra "textualidad nacional" previamente existente, enemiga y finalmente vencida, la de la España republicana ${ }^{10}$. Uno de los principales reproches que usó el franquismo para desacreditar la actividad cultural llevada a cabo durante la República fue precisamente su presunto carácter extranjerizante y "bastardo".

\footnotetext{
${ }^{8}$ La denominada Ley Fraga (1966) supone un hito visible en los cambios sufridos por el aparato censor franquista a lo largo de su existencia, pero los estudios actuales recogen periodizaciones acordes a los cambios políticos experimentados por el régimen: sin duda la más desarrollada corresponde a Ruiz Bautista (2008); pero cabe recordar a Jeroen Vandaele (2010), o en relación a las traducciones al catalán las propuestas en Coll-Vinent, Eissner, Gallén (2011).

${ }^{9}$ En el año 1958, el censor rechazaba la publicación de una obra de Bertolt Brecht con el argumento: "[...] Brecht es un autor conocido por su actitud pro-soviética y por su pasado y sus tendencias netamente comunistas, opinamos que debe PROHIBIRSE LA PUBLICACIÓN DE ESTA OBRA" (AGA, caja 21/12132, expediente 4375). En el año 1964 escribe otro censor: "A pesar de las conocidas tendencias izquierdistas del autor, estimamos razonable este tipo de crítica. PUEDE AUTORIZARSE (AGA, caja 21/15908, expediente 4204)".

${ }^{10}$ Ana María Martínez Rus (2008), entre otras muchísimas, ofrece esta cita de Antonio Luna, secretario nacional de la Jefatura de Educación, hecha el 2 de mayo de 1939 en un acto universitario: "Para edificar a España una, grande y libre, condenados al fuego los libros separatistas, los liberales, los marxistas, los de la leyenda negra, los anticatólicos, los del romanticismo enfermizo, los pesimistas, los pornográficos, los de un modernismo extravagante, los cursis, los cobardes, los seudocientíficos, los textos malos y los periódicos chabacanos". La depuración de libros en catalán durante el primer franquismo está estudiada a fondo en Gallofré (1991).
} 
Otro reproche obsesivo presente en las diatribas franquistas contra la realidad cultural republicana fue el de la permisividad dada a las llamadas manifestaciones culturales separatistas y disgregantes, es decir, a la producción cultural en catalán, euskera o gallego. Poco integrado (o ni siquiera mencionado) en los trabajos globales que se ocupan del estudio de la censura y la traducción en España, abordado si acaso en capítulos a parte, el tratamiento que la censura dispensó a la actividad editorial y traductora realizadas en catalán, en euskera y en gallego durante el franquismo representa, a mi modo de entender, un aspecto esencial de la "textualidad nacional" franquista, puesto que define muy bien su relación con unas realidades culturales vivas que no pudiendo ser consideradas extranjeras, fueron vistas, con gran menosprecio y de forma muy hostil ${ }^{11}$, como realidades ajenas e indeseables que convenía reprimir y reducir como mucho a lo folklórico.

Levantado el veto a las traducciones al catalán a principios de los años $60^{12}$, todavía es posible en el año 1964 leer en el expediente censor $^{13}$ a la solicitud de publicación de la traducción catalana de la novela de Marcel Proust Un amor de Swan las siguientes palabras:

Proust no es un autor, no digo ya de masas, ni casi de minorías amplias; no quiero decir que no sea un gran escritor. Pero no es fácilmente soportable hoy. Es difícil seguir el hilo argumental. Como todas sus obras la ambienta en una determinada sociedad, alta parisina y sobre una levísima armazón narradora, reuniones, visitas, fiestas, amores, etc. Va exponiendo su pensamiento, su filosofía sobre los diversos temas. Filosofía la mayoría de las veces no ortodoxa, en muchos casos, y para algunos lectores, perjudicial. Pero nosotros solo debemos actuar en peligro común. Quiero decir que a este autor hay que mirarlo con la escopeta baja y el seguro echado. ¿Para qué gastar pólvora? Y se [sic] va a un dialecto, mejor que mejor. PROCEDE SU AUTORIZACIÓN.

Finalmente cabe hablar de la marcada opacidad que dominó la institución censora española -independiente de los organigramas organizativos-, como cabe inferir del estudio de algunos expedientes de censura que hallamos en el AGA, pero también de las experiencias relatadas

\footnotetext{
${ }^{11}$ Montserrat Bacardí (2012: 11-94).

${ }^{12}$ Jordi Cornellà-Detrell (2013: 47-67).

${ }^{13}$ AGA caja 21/15182, expediente 2478 .
} 
principalmente por los editores de la época en obras de carácter autobiográfico ${ }^{14}$. La remisión de una obra al aparato censor por parte de los editores (hasta el año 1966 obligatoria, después de ese año más que recomendable) supuso en numerosas ocasiones solo el primer paso en el largo y tortuoso recorrido antes de su publicación, de hacerse efectiva. Con suerte para el investigador, la documentación conservada nos ayuda a reconstruir esos complicados vericuetos ${ }^{15}$. Otras veces la documentación hallada es nula, escueta, insuficiente o contradictoria con lo que realmente ocurrió ${ }^{16}$. En cualquier caso, el conocimiento de esta documentación es indispensable para la reconstrucción de los principios de "textualidad nacional" que rigieron las actuaciones censorias. Aunque quepa recordar que la autorización de una obra se resolvía a menudo en visitas a ciertos despachos ${ }^{17}$.

El término propuesto por Francesca Billiani, a pesar de los problemas mencionados en su aplicación, nos remite sea como sea a la necesidad de llenar de contenido concreto los criterios con los que actuó la censura al valorar las traducciones (¿Ataca a la Moral?, etc.). Dada la inexistencia de un decálogo de principios definidos, la aplicación de la noción "textualidad nacional" al análisis de la documentación generada por el aparato censor, con sus debidas puntualizaciones, puede aportar algo de claridad a una realidad oscura y mutante.

\section{2. "Censura/ convenciones literarias/ buen gusto"}

Los parámetros de descripción textual que sugieren Rundle y Sturge en su estudio Translation under Fascism resultan muy pertinentes para el análisis de las alteraciones llevadas a cabo en textos traducidos y

\footnotetext{
${ }^{14}$ Barral (2001), Castellet (2012).

${ }^{15}$ Las andaduras que siguieron las solicitudes para la publicación de la novela de Günter Grass Die Blechhtrommel (El tambor de hojalata), tramitadas por Carlos Barral en 1959 primero para la traducción al castellano (AGA, caja 21/12590, expediente 5043) y por la editorial Aymà para la traducción al catalán en 1966, son dignas de un thriller político, como estudié en Jané-Lligé (2013).

${ }^{16}$ A diferencia del caso anterior, los trámites de autorización a la solicitud de publicación de otra novela de Günter Gras Katz und Maus (El gato y la rata) por Seix Barral, siendo inicialmente positivos, no concluyen con la edición de la obra sin que en la documentación conservada podamos hallar la razón (Jané-Lligé, 2015).

${ }^{17}$ Castellet (2012: 29-34).
} 
publicados bajo la amenaza de la censura, concretamente en regímenes fascistas. Los autores convienen que cabría establecer una distinción conceptual clara entre las alteraciones textuales debidas a la intervención del aparato censor de aquellas otras que fueron fruto del respeto por parte de traductores y editores a determinadas convenciones literarias vigentes o a normas consideradas de "buen gusto" (Rundle \& Sturge, 2010: 7). Esta distinción, a mi modo de ver muy relevante, reclama un mayor acercamiento a los textos traducidos, más sistemático y más sutil, que debería formar parte de cualquier estudio de recepción, dado que son los textos traducidos -más o menos alterados- los textos que llegaron al público lector.

El análisis de la recepción de las primeras obras de Günter Grass en España ilustra de forma diáfana la pertinencia de esta clarificación de criterios. A pesar de haber visto previamente rechazada por la censura la publicación de su obra El tambor de hojalata, en los años 1968 y 1973 vieron la luz respectivamente las traducciones catalana y castellana de su segunda novela, Katz und Maus ${ }^{18}$.

El análisis minucioso de ambas traducciones ${ }^{19}$ nos permite atribuir algunas de las alteraciones presentes en el texto a decisiones tomadas por el editor o el traductor y no a las exigencias hechas por los censores $^{20}$, que incidían especialmente en cuestiones de moral y "buen gusto" y que fueron obviamente satisfechas. En lo relativo a las convenciones literarias, cabe constatar tanto en la traducción catalana como en la castellana una fuerte tendencia a la homogeneización estilística del texto hacia un registro lingüístico muy formal y "literario", hecho que conlleva una neutralización de todas las modulaciones de registro que tienen lugar en el texto original y que son una de las características esenciales del estilo de Grass. Así, el recurso a los dialectos geográficos, de los que se sirve el autor para caracterizar a algunos personajes de la obra (la madre y la tía del protagonista se expresan a menudo en el dialecto de Danzig), o bien al registro coloquial, que caracteriza entre otros a los jóvenes soldados del ejército alemán, pierde en ambas traducciones

\footnotetext{
${ }^{18}$ La traducción catalana, de Carles Unterlohner, fue publicada por Edicions 62. La traducción castellana, de Carlos Gerhard, por Barral Editores.

${ }^{19}$ Jané-Lligé (2015).

${ }^{20}$ Esas exigencias quedan recogidas en sendos expedientes: 1) para la traducción catalana, en AGA caja 21/18098, expediente 3457; 2) para la traducción castellana, en AGA caja 21/14280, expediente 6447 .
} 
toda la fuerza expresiva que tiene en el original. Veamos un fragmento en el que un joven oficial alemán describe de forma vivaz a un auditorio formado por jóvenes bachilleres las persecuciones aéreas de las que fue protagonista:

[...] nun müsst ihr nicht denken, das läuft wie ne Karnickenjagd, mit drauf und los und hastjenichjesehen. Oft wochenlang nichts. Aber als wir an den Kanal - dacht ich, wenn hier nicht, dann nirgends. Und es klappte (Grass, 1961: 61).

El texto reproduce elisiones fonéticas ([ei]ne, dacht $[\mathrm{e}]$ ), elisiones de conjunciones (...denken, [dass] das läuft...) y de verbos (...wenn hier nicht [etwas passiert], dann nirgends...) muy propias de la lengua hablada; el vocabulario usado por el narrador es muy coloquial (Karnickenjagd, klappte) y este recurre a menudo a juegos de palabras (hastjenichjesehen) y expresiones populares (mit drauf und los). Todos estos elementos se ven neutralizados en la traducción castellana (algo parecido ocurre en la catalana):

[...] bien muchachos, no vayáis a creer ahora que esto es como una cacería de conejos, atacar, echarse a un lado y volver a atacar todo el tiempo. A veces nada ocurre por espacio de varias semanas. Pero cuando fuimos al Canal... bueno, me dije para mí, ahora o nunca. Y efectivamente, así fue [...] (Grass, 1973: 60).

Otros elementos habituales de la prosa de Grass, como la experimentación con la forma, se ven a menudo reducidos en las traducciones a la mínima expresión ${ }^{21}$. Asimismo, cabe atribuir a las convenciones literarias del momento la traducción al castellano del nombre del protagonista de la novela: de este modo Joachim se convierte en Joaquín.

Los criterios propuestos por Rundle y Sturge para el análisis de las traducciones contribuyen sin duda a mejorar nuestro análisis textual y a profundizar en el conocimiento de las convenciones literarias imperantes en España durante el franquismo.

\footnotetext{
${ }^{21}$ Jané-Lligé (2015).
} 


\section{Los editores y la traducción: Josep Janés, Carlos Barral y Josep Maria Castellet}

Pero si para la descripción de las características de las traducciones editadas en España bajo el franquismo es imprescindible dotarse de herramientas que describan minuciosamente la acción oficial y gubernamental así como sus efectos en los textos traducidos, no es de menor importancia abordar con detalle el quehacer de los que fueron responsables de canalizar mayormente la actividad traductora en ese período histórico: los editores y la iniciativa privada autorizada. Josep Janés, Carlos Barral y Josep Maria Castellet dedicaron buena parte de sus esfuerzos a publicar literatura traducida bajo el franquismo, los dos primeros al castellano y el tercero al catalán. Sus catálogos editoriales pretendieron ofrecer, en la medida en que se lo permitieron, un discurso cultural alternativo al discurso de la oficialidad y fueron sin duda referentes indiscutibles para las nuevas generaciones de escritores y para el nuevo público. En este sentido, escribe José Carlos Mainer en el prólogo al estudio de Jacqueline Hurtley (1992) sobre la labor de Janés como editor de literatura inglesa:

No es casual que la primera novela de Carmen Laforet tuviera -en su ingenuidad- tanto de romanticismo gótico como de aire británico. O que un narrador premiado por Janés con el Premio Internacional -me refiero a Ildefonso Manuel Gil, que lo ganó con La moneda en el suelo (1949)- confesara en una entrevista que su autor predilecto era Charles Morga, uno de los puntales de la editorial. O que el joven Ricardo Gullón dedicara un libro, Novelistas ingleses contemporáneos (1945), al estudio de quienes -desde Thomas Hardy y Joseph Conrad hasta Maurice Baring, Rosamond Lehman o Margaret Kennedy- se vieron en las palabras españolas gracias al esfuerzo de nuestro editor (Mainer, 1992: 19).

Es imposible entender la naturaleza de las iniciativas editoriales de estos tres importantes editores en el panorama editorial español, a cuya renovación contribuyeron de forma muy remarcable, sin tener en cuenta sus estrechos vínculos con la industria editorial barcelonesa prefranquista y con la edición en catalán. Lo señala Jacqueline Hurtley para los catálogos de Janés, describiéndolos en buena medida como continuación de su ambicioso proyecto editorial noucentista en cata- 
lán ${ }^{22}$, no exento de una clara intencionalidad política en el marco del franquismo. También alude a este hecho Carlos Barral en sus textos autobiográficos al hablar de sus más estrechos colaboradores literarios en la editorial Seix Barral: el "sabio" Joan Petit ${ }^{23}$ o el mismo Josep Maria Castellet. En lo siguiente quisiera ilustrar diferentes aspectos de la labor de estos editores que ayudan a reconstruir las coordenadas en que tuvieron que moverse.

\subsection{Josep Janés y la literatura no inglesa}

Al lado de su díscola labor de divulgación de la literatura inglesa, con intención de ofrecer un discurso cultural liberal y alternativo (Hurtley, 1992: 222), Josep Janés también supo y tuvo que contentar al régimen con la publicación de autores más afines a la ideología dominante. Así aparecen publicados en sus catálogo ${ }^{24}$ autores abiertamente simpatizantes con el fascismo, como Knut Hamsun ${ }^{25}$, o autores alemanes que no cayeron en desgracia bajo el nazismo, como Hans Fallada ${ }^{26}$. Atento a los vaivenes ideológicos del régimen, el editor barcelonés supo hacerse con fondos editoriales del pre-franquismo que reutilizó en su momento adecuado. Así, Janés publicó algunas de las traducciones de las más importantes obras de Thomas Mann ${ }^{27}$ que ya habían circulado durante la República y que entre 1939 y 1945 habían desaparecido del mercado, hecho que hay que atribuir a la postura manifiestamente beligerante de Mann contra el régimen de Hitler ${ }^{28}$. En cambio, entre 1945 y 1947

\footnotetext{
${ }^{22}$ Hurtley (1986).

${ }^{23}$ Barral (2001: 310-313).

${ }^{24}$ Hurtley (1992); Josep Mengual (2013).

${ }^{25}$ Las solicitudes que Janés presentó a censura para la publicación de obras de Hamsun fueron siempre favorables y nada problemáticos: Pan (1940) (AGA caja 21/06580 expediente 194; Victoria (1941) (AGA caja 21/06697, expediente 520).

${ }^{26}$ Janés publicó cuatro obras de este autor en los años 40 sin ningún tipo de obstáculo administrativo: Corazón viejo a la aventura (1943); Una vez tuvimos un hijo (1944); El hombre que no fue amado (1944); Gustavo de férreo (1947).

${ }^{27}$ Es el caso de la traducción de La montaña mágica, de Mario Verdaguer, publicada en 1934.

${ }^{28}$ En 1939 se presentó a censura la obra Mario y el encantador (AGA, caja 21/06458 expediente 464), obteniendo autorización pero no llegando a ser publicada. Entre 1939 y 1945 no existe en el AGA ninguna solicitud de publicación para una obra de Mann. A diferencia de lo que ocurrió con los escritores judíos alemanes (Ruiz Bautista, 2008: 57).
} 
encontramos en los archivos del AGA nada menos que seis solicitudes de publicación de obras de Thomas Mann, todas ellas con resolución favorable, salvo La muerte en Venecia ${ }^{29}$. El informe a la traducción de Mario Verdaguer de La montaña mágica, escrito por el poeta Leopoldo Panero, dice lo siguiente:

La obra se desarrolla y tiene como fondo un sanatorio antituberculoso enclavado en los Alpes suizos. Es generalmente considerada como una de las creaciones imaginativas más sólidas y grandiosas de la moderna novelística. El espíritu que se desprende de sus páginas y que es como la resultante moral e intelectual de sus personajes es profundamente humano y está impregnado de poesía y de ternura. No creemos desde luego que ofrezca ningún peligro, máxime teniendo en cuenta el restringido público a que se dirige por su especial carácter. Juzgamos por lo tanto oportuna su aprobación ${ }^{30}$.

\subsection{Carlos Barral, autor de epístolas a Censura}

La labor de Carlos Barral se circunscribe en una etapa posterior del franquismo, cuando este se encontraba en una fase de estabilidad económica y pretendía la pacificación social. La inquieta labor del editor barcelonés se ha de relacionar con su conocimiento y contactos con otros prestigiosos editores europeos del momento (Castellet, 2011: 20), responsables de catálogos comprometidos con la acción del intelectual en la sociedad. Las propuestas editoriales de Barral rayaron a menudo la provocación, como demuestran los expedientes censorios conservados, que nos aportan documentación muy valiosa. Especialmente interesantes para el conocimiento de la censura y las presiones a que los editores intentaban someterla, puesto que vemos en ellas qué tipo de argumentos usó Barral, son las cartas suplicatorias que el editor barcelonés envió a los censores para intentar alterar su veredicto negativo acerca de una solicitud de publicación, ya se tratase de la total prohibición o bien de la exigencia de supresión o modificación de fragmentos. He aquí un ejemplo de carta de desacuerdo ante la negativa de los censores a la publicación de Opiniones de un payaso, del novelista alemán Heinrich Böll:

\footnotetext{
${ }^{29}$ AGA, caja 21/07997, expediente 2167.

${ }^{30}$ AGA caja 21/05709 expediente 5923.
} 
CONSIDERANDO que Heinrich Böll es considerado por la crítica alemana e internacional como uno de los más altos representantes de la narrativa católica contemporánea, y no por el hecho de que Heinrich Böll sea católico sino porque su obra tiene, como la de Graham Greene, una evidente intención moralizadora y parte de una concepción confesional. CONSIDERANDO que el hecho de que la obra de un novelista católico como Heinrich Böll no pueda publicarse en España por razones de Censura resultará a los ojos de la gente de letras de todo el mundo totalmente incomprensible y que la difusión de esta noticia es inevitable tratándose de uno de los más importantes narradores europeos contemporáneos.

SUPLICA que le sea concedida la autorización para editar una traducción española de dicho libro ${ }^{31}$.

La novela se acabó publicando en 1965.

\subsection{Josep Maria Castellet i la renovación del mundo editorial en catalán}

La labor de Josep Maria Castellet al frente de Edicions 62 es emblemática, junto con la de otros editores catalanes contemporáneos, de la clara voluntad de los editores y el público catalán de no ver reducida su cultura a expresión folklórica o nostálgica. El esfuerzo de traducción y supervivencia cultural hecho en Cataluña desde los años sesenta hasta el final del franquismo ${ }^{32}$, con el catalán ausente y completamente prohibido en la oficialidad, en la escuela, en los medios de comunicación de masas, es sin duda un fenómeno sociolingüístico digno de ser conocido y estudiado. He aquí un anuncio de la editorial Edicions 62 en la revista Serra d'Or que ilustra muy bien el carácter internacionalista de estas iniciativas:

La col·lecció de novel·les més representativa de tots els corrents mundials d'avui: EL NOVEAU ROMAN FRANCĖS, LA GRAN LITERATURA DELS ESTATS UNITS, LA MODERNA LITERATURA ALEMANYA, EL NEOREALISME ITALIÀ, L'ESTABLISHMENT

\footnotetext{
${ }^{31}$ AGA, caja 21/14942 expediente 144.

${ }^{32}$ Coll-Vinent, Eissner \& Gallén (2011); Bacardí (2012).
} 
LITERARI ANGLÈS, I ELS ACTUALS NOVEL·LISTES CATALANS $^{33}$.

Efectivamente, el catálogo de Edicions 62 de estos años no tiene nada que envidiar al de cualquier gran editorial europea de esa época.

\section{Conclusiones}

La vida editorial y literaria de la España franquista estuvo en buena medida definida por el tira y afloja entre las iniciativas editoriales de carácter privado y las actuaciones institucionales de la censura. La traducción supuso una parte importante de esa actividad editorial. El estudio de la historia de las traducciones publicadas (y de las prohibidas) en España durante ese período contribuye a arrojar luz sobre un período todavía oscuro en muchos aspectos. El análisis del trato que la censura dio a esas iniciativas nos dice mucho de los principios estéticos e ideológicos que rigieron el aparato censor y, por extensión, las políticas de los diferentes gobiernos franquistas y que, en ningún caso, fueron ajenos a las vicisitudes políticas internas y externas por las que atravesó el régimen. El análisis textual detallado de las traducciones debe contribuir también a determinar de un lado de qué modo fueron leídas las literaturas extranjeras y qué principios estéticos cambiantes dominaron en España durante el régimen franquista.

\section{Bibliografía}

Abellán, Manuel L. 1980. Censura y creación literaria en España (19391976). Barcelona: Península.

Barral, Carlos. 2001. Memorias. Barcelona: Península.

Billiani, Francesca. 2007. Modes of Censorship and Translation: National Contexts and Diverse Media. Mánchester: St. Jerome.

Castellet, Josep Maria. 2001. La memoria de Carlos Barral. En Barral, Carlos (ed.) Memorias. Barcelona: Península, 9-10.

Castellet, Josep Maria. 2012. Memòries confidencials d'un editor. Barcelona: Edicions 62.

Coll-Vinent, Sílvia; Eissner, Conelia \& Gallén, Enric (coord.). 2011. La traducció $i$ el món editorial de postguerra. Lleida: Punctum \& Triclat.

\footnotetext{
${ }^{33}$ Anuncio publicado en el número de abril de 1965 de la revista Serra d'Or.
} 
Cornellà-Detrell, Jordi. 2013. L'auge de la traducció catalana als anys 60: el desglaç de la censura, el XVI Congreso de Editores I el problema dels drets d'autor. Quaderns. Revista de traducció 20, 47-67.

Gallofré, Maria Josepa. 1991. L'edició catalana i la censura franquista (19391951). Barcelona: Publicacions de l'Abadia de Montserrrat.

Gili Roig, Gustavo. 1944. Bosquejo de una política del libro. Barcelona: Gustavo Gili Roig.

Gracia, Jordi \& Ruiz Carnicer, Miguel Ángel. 2001. La España de Franco (1939-1975) Cultura y vida cotidiana. Barcelona: Síntesis.

Grass, Günter. 1961. Katz und Maus. Neuwies: Luchterhand.

Grass, Günter. 1968. El gat i la rata. Barcelona: Edicions 62.

Grass, Günter. 1973. El gato y el ratón. Barcelona: Barral Editores.

Hurtley, Jacqueline A. 1986. El combat per la cultura. Barcelona: Curial.

Hurtley, Jacqueline A. 1992. José Janés: Editor de literatura inglesa. Barcelona: PPU.

Inguilleri, Moira. 2005. The sociology of Bourdieu and the construction of the "object" in translation and interpreting studies. The Translator 11(2): 125-145.

Jané-Lligé, Jordi. 2011. Heinrich Böll, Günter Grass i Wolfgang Koeppen i el món editorial de postguerra. En Coll-Vinent, Sílvia; Eissner, Cornelia \& Gallén, Enric (coord.) La traducció i el món editorial de postguerra. Lleida: Punctum \& Triclat, 95-110.

Jané-Lligé, Jordi. 2013. Narrativa alemanya de postguerra: autors traduïts i censura. Quaderns. Revista de traducció 20: 118-145.

Jané-Lligé, Jordi. 2015. Literary translation and censorship: a textual approach. En Bączkowska, A. (ed.) Perspectives on Translation. Newcastle: Cambridge Scholars. Series: Studies in Language and Translation.

Mainer, José Carlos. 1992. Prólogo. En Janés, José (ed.) Editor de literatura inglesa. Barcelona: PPU, 18-20.

Martínez Cachero, José María. 1997. La novela española entre 1936 y el fin de Siglo: Historia de una aventura. Madrid: Castalia.

Martínez Rus, Ana María. 2013. Expolios, hogueras, infiernos. La represión del libro. Represura 8. doi: http://www.represura.es/represura_8_febrero_2013_articulo2.html.

Mengual, Josep. 2013. A dos tintas. Josep Janés, poeta y editor. Barcelona: Debate.

Muñoz Lloret, Teresa. 2006. Josep M. Castellet. Retrat de personatge en grup. Barcelona: Edicions 62.

Post, Robert C. 1998. Censorship and Silencing: Practices of Cultural Regulation. Los Ángeles: The Getty Research Institut. 
Prunč, Erich. 2012. Entwicklungslinien der Translationwissenschaft. Berlín: Frank \& Time.

Ruiz Bautista, Eduardo. 2005. Los señores del libro: propagandistas, censores $y$ bibliotecarios en el primer franquismo. Gijón: Ediciones Trea.

Ruiz Bautista, Eduardo (coord.). 2008. Tiempo de censura. La represión cultural durante el franquismo. Gijón: Ediciones Trea.

Rundle, Christopher \& Sturge, Kate. 2010. Translation under Fascism. Houndmills: Palgrave Macmillan.

Sanz Villanueva, Santos. 2010. La novela española durante el franquismo. Madrid: Gredos.

Seruya, Teresa \& Lin Moniz, Maria. 2008. Translation and Censorship in different times and landscapes. Newcastle: Cambridge Scholars Publishing.

Vandaele, Jeroen. 2010. It was what it wasn't: Translation and Francoism. En Christopher, Rundle \& Sturge, Kate (eds.) Translation Under Fascism. Houndmills: Palgrave Macmillan, 84-117.

Ysàs, Pere (2004). Disidencia y subversión: la lucha del régimen franquista por su supervivencia, 1960-1975. Barcelona: Crítica. 